\title{
Monolith formation and ring-stain suppression in low-pressure evaporation of poly(ethylene oxide) droplets
}

\author{
Kyle A. Baldwin ${ }^{1}$, Samuel Roest ${ }^{1}$, David J. Fairhurst ${ }^{1} \uparrow$, Khellil Sefiane $^{2}$ \\ and Martin E. R. Shanahan ${ }^{3}$ \\ ${ }^{1}$ School of Science and Technology, Nottingham Trent University, Nottingham NG11 8NS, UK \\ ${ }^{2}$ School of Engineering, University of Edinburgh, Edinburgh EH9 3JL, UK \\ ${ }^{3}$ Université de Bordeaux, Institut de Mécanique et d'Ingénierie-Bordeaux (UMR 5295), Bâtiment A4, \\ 351, Cours de la Libération, 33405 Talence CEDEX, France
}

(Received 26 September 2011; revised 25 November 2011; accepted 5 January 2012)

When droplets of dilute suspensions are left to evaporate the final dry residue is typically the familiar coffee-ring stain, with nearly all material deposited at the initial triple line (Deegan et al., Nature, vol. 389, 1997, pp. 827-829). However, aqueous poly(ethylene oxide) (PEO) droplets only form coffee-ring stains for a very narrow range of the experimental parameters molecular weight, concentration and drying rate. Instead, over a wide range of values they form either a flat disk or a very distinctive tall central monolith via a four-stage deposition process which includes a remarkable bootstrap-building step. To predict which deposit will form, we present a quantitative model comparing the effects of advective build-up at the triple line to diffusive flux and use this to calculate a dimensionless number $\chi$. By experimentally varying concentration and flux (using a low-pressure drying chamber), the prediction is tested over nearly two orders of magnitude in both variables and shown to be in good agreement with the boundary between disks and monoliths at $\chi \approx 1.6$.

Key words: condensation/evaporation, drops, polymers

\section{Introduction}

Although a metaphor for boredom, there has been a growing scientific interest over the last decade in watching droplets of paint (and other liquids) dry. The subtle interplay between surface forces and bulk behaviour provides intellectual intrigue, and the widespread applications (e.g. inkjet printing and cooling technologies) demand commercial attention. In the seminal work of Deegan et al. (1997) the common occurrence of 'ring stains' from spilt coffee is explained due to outwards radial flow within the evaporating droplet. However, the final morphology of dried suspensions is not always a ring but depends on many experimental factors including: the solvent evaporation rate (Marín et al. 2011); particle shape (Yunker et al. 2011); self-assembly and organization as mediated by solvent dewetting (Stannard 2011); interactions between solvent, vapour and substrate (Rowan et al. 2000; Li \& Graf 2009); phase

$\dagger$ Email address for correspondence: david.fairhurst@ntu.ac.uk 
transitions within the droplet (Parisse \& Allain 1997; Pauchard \& Allain 2003); and the contribution of convection currents (Hu \& Larson 2006).

In addition to suspensions, drying experiments have been performed on droplets of polymer solutions. Pauchard and Allain observed that during evaporation of dextran (a branched polysaccharide), the concentration at the surface increases until a glassy skin forms (Pauchard \& Allain 2003). They proposed that the skin is water permeable yet incompressible so that upon further evaporative volume loss the constant-area skin is subjected to stress and buckles leading to various final shapes, including doughnutand sombrero-like deposits, which are predicted from initial values of contact angle, humidity, temperature and concentration.

Poly (ethylene oxide), or PEO, is a very common and widely used linear polymer, unusual amongst its homologues for its good solubility (Hammouda, Ho \& Kline 2004). It dissolves in water, although at high concentrations or molecular weights solutions can appear cloudy due to micron-sized clusters of undissolved polymer (Hammouda et al. 2004). Recent experimental work on the evaporation of PEO droplets (Willmer et al. 2010; Baldwin et al. 2011) proposed a four-stage drying mechanism including a 'bootstrap' stage in which the liquid droplet is lifted above the surface by the freshly formed solid deposit, resulting in solid monoliths. These structures, which can be taller than the initial droplet, clearly defy the typical ring-stain effect, as nearly all the dried material ends up in the centre, rather than distributed around the edge of the initial triple line (where the three materials, liquid droplet, vapour and solid substrate all meet). Despite widespread use of the polymer, the seemingly unique drying behaviour of droplets of PEO is a recent discovery and understanding of the mechanisms determining the formation of the unusual deposits is currently incomplete: one prediction is that monoliths are more likely to form in droplets with high initial concentration $c_{0}$ or high evaporation rate $-\dot{V}$, where $V$ is the droplet volume. Only for droplets with very low initial concentrations are ring-stain deposits seen.

In this work we consider solvent and polymer dynamics within a thin annulus at the edge of the droplet to develop a semi-quantitative model to predict the formation of monoliths. The predictions agree well with previously published data (Baldwin et al. 2011), and with data from approximately 50 new experiments expanding the range of $\dot{V}$ values to cover almost two orders of magnitude, by reducing ambient pressure within the drying chamber. These low-pressure observations also offer suggestions as to the underlying mechanism.

\section{Materials and methods}

Solutions were prepared using $\mathrm{PEO}$ with an average molecular weight $M_{w} \approx$ $100 \mathrm{kDa}$ spanning a range of initial mass fractions $c_{0}$ from 0.01 to 0.35 and were mixed using distilled, deionized water and left to equilibrate for at least $24 \mathrm{~h}$, and inverted several times to ensure uniform viscosity before use. For each measurement, a droplet with initial volume $V_{0}=10 \mu \mathrm{l}$ was slowly pipetted onto a clean glass microscope coverslip. The coverslip was placed into a cylindrical lowpressure chamber (diameter $8.6 \mathrm{~cm}$, height $5.4 \mathrm{~cm}$ ), connected to either a Cole Parmer diaphragm pump (for pressures $P$ down to 25 mbar) or an Edwards oil diffusion pump (for pressures down to $0.6 \mathrm{mbar}$ ), and a pressure sensor giving precise manual control of the pressure after droplet deposition. A plastic baffle was placed within the chamber to reduce the effect of net air flow on the drying droplet. The droplet was illuminated by a diffuse light source, placed behind the droplet outside the chamber, 


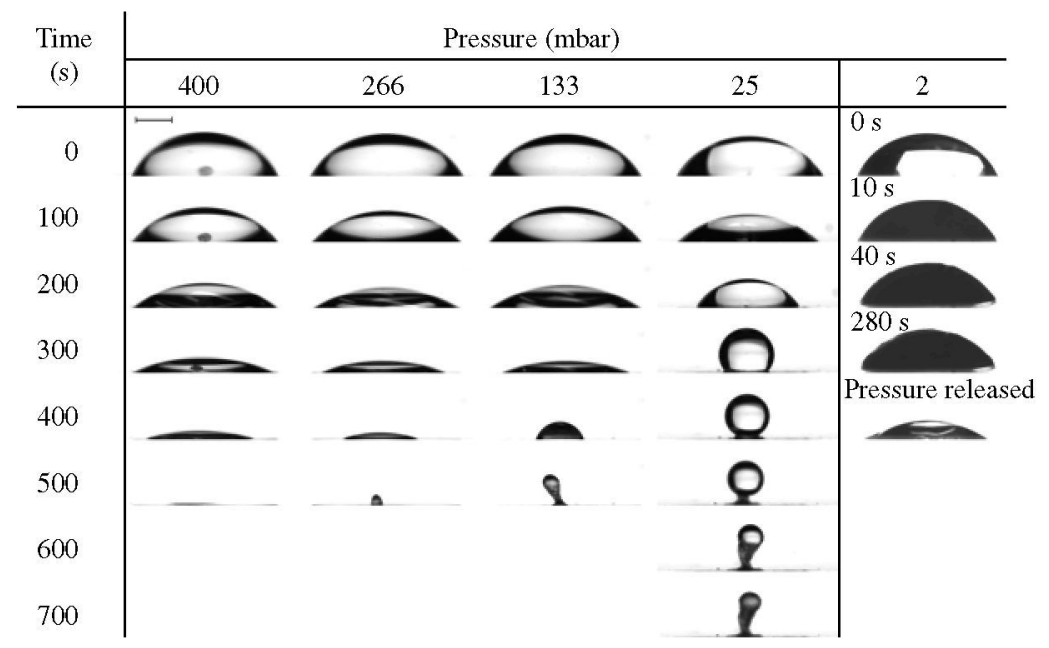

FIGURE 1. Time-lapse images with time increasing in $100 \mathrm{~s}$ intervals downwards, showing, in the first four columns, behaviour for a range of $10 \mu \mathrm{l}$ droplets with initial concentration $c_{0}=0.02$ and decreasing pressures (from left to right $P=400,266,133,25 \mathrm{mbar}$ ). In the far right column the pressure is sufficiently low $(2 \mathrm{mbar})$ that the outer layer of the droplet freezes, which if left under vacuum would continue to sublime. When the vacuum is released, as illustrated in the fifth image of the far right column, the frozen crust melts. The scale, indicated by the $1 \mathrm{~mm}$ scale bar in the upper left, is the same for all images. Movies 1 and 2 show this behaviour for two additional droplets at $P=25 \mathrm{mbar}$ with $c_{0}=0.015$ and 0.05 , respectively, available with the online version of this paper at journals.cambridge.org/flm.

and a digital camera recorded images of the drying process every second. To prevent dissolved air forming disruptive bubbles within the evaporating droplets, all solutions were initially degassed at 50 mbar for around $30 \mathrm{~s}$ until bubbling ceased. Droplets drying at atmospheric pressure were prepared and recorded as described in Baldwin et al. (2011).

Under atmospheric conditions, $\dot{V}$ can be determined both by gravimetric means and by fitting the acquired droplet images with a spherical cap to determine the volume evolution. However, at low pressures neither of these methods is satisfactory: the mass balance cannot be used under vacuum and solid deposits occur almost immediately, distorting the spherical shape of the droplet, before sufficient measurements of $V$ can be taken. Instead we use $10 \mu 1$ droplets of pure water at the same pressure to determine $\dot{V}$ and assume that the polymer concentration does not significantly affect evaporation rate: in previous work (Baldwin et al. 2011) it was found that the evaporation rate was affected noticeably by relative humidity $R H$, droplet size (characterized by the radius of the circular contact area $R$ ), temperature $T$ and ambient pressure via $\dot{V} \propto R T(1-R H) / P$ Cazabat \& Geoffroy 2010), but not by PEO concentration. However, to account for inaccuracies in using this assumption, we introduce $30 \%$ uncertainty in values of $\dot{V}$.

\section{Results and discussion}

Figure 1 shows five sequences of images for $c_{0}=0.02$ and values of $P$ decreasing from left to right, with movies 1 and 2 showing more detail of two additional droplets, both at $P=25$ mbar with $c_{0}=0.015$ and 0.05 , respectively. All monolith-forming droplets show the same four stages of drying as have been previously reported 
(Baldwin et al. 2011). Initially, the triple line (TL) is pinned to the substrate, giving drying with constant contact area between solid and liquid. During the second stage the triple line recedes through pseudo-dewetting: it is not strictly dewetting, as a thin solid deposit is left behind. Unusually, the contact angle $\theta$ (measured between the liquid and the solid at the TL) increases, as can be seen by comparing the second and third images for $P=25$ mbar in figure 1 ; typically when a liquid interface recedes, $\theta$ remains constant. In the third stage the liquid droplet is lifted upwards by solid precipitate, in a stage referred to as 'bootstrap' growth. This can be seen very clearly in the fifth and sixth images for $P=25 \mathrm{mbar}$ in figure 1 . The final stage (shown in the accompanying movies) is a slow shrinking of the solid deposit as remaining water evaporates, during which there is no significant shape change. Depinning is seen as an essential prerequisite to monolith formation, as the solid deposit must follow the footprint of the drop. If the drop remains pinned, then it forms a puddle; if the drop depins, the footprint shrinks and thus the final deposit is a monolith.

It is clear from the images in figure 1 that, as expected, the initial evaporation rate is faster at lower pressures: the droplet drying at $P=25$ mbar is noticeably lower after $100 \mathrm{~s}$, and depins and is receding at $200 \mathrm{~s}$, earlier than any others. (Depinning occurs as crystallization begins at the TL, which will occur earlier when evaporation rate is higher.) Intriguingly, though, from this point onwards, low-pressure droplets evaporate more slowly. This may be due to several factors, including: formation of a polymer skin, which inhibits evaporation, and the known dependence of evaporation rate on droplet contact radius $\dot{V} \propto R$ (Cazabat \& Geoffroy 2010). Follow-up work is clearly required to fully investigate this experimental observation.

At lowest pressures $(P=2 \mathrm{mbar})$ as evaporative cooling removes heat from the droplet, the outer layer of the droplet freezes into a crust (as shown in the final column of figure 1). When the valve is opened and the chamber returns to atmospheric pressure, the frozen crust quickly melts. By balancing latent heats of vaporization $\left(L_{v} \approx 2500 \mathrm{~J} \mathrm{~g}^{-1}\right)$ and fusion $\left(L_{f} \approx 330 \mathrm{~J} \mathrm{~g}^{-1}\right)$ and heat capacity for water $\left(C \approx 4.2 \mathrm{~J}(\mathrm{gK})^{-1}\right)$, and assuming no heat flow from the environment, the fraction of a droplet at initial temperature $\Delta T$ above freezing which would need to evaporate in order to remove sufficient heat for the entire remaining droplet to freeze is $1-L_{v}\left(L_{v}+L_{f}+C \Delta T\right)^{-1} \approx 15 \%$. The droplets observed here do not freeze entirely, only forming a frozen crust, so should require a smaller volume loss, in line with our qualitative observations. Crust freezing disrupts the four-stage process and introduces a lower/upper limit to the pressure/evaporation rate at which we should expect monoliths to form.

Figure 2 is a phase diagram showing, as a function of the experimental parameters $c_{0}$ and $P$, a profile image of dried structures: this is not an exhaustive representation of all data, but a selection of 20 representative images chosen from the 50 new experiments to indicate the dependence on the two experimental parameters $c_{0}$ and $P$. As expected, lower concentration droplets leave smaller deposits, but also require lower pressures to achieve monoliths. Higher $c_{0}$ leads to earlier PEO deposition and therefore larger final structures. These larger structures are much more likely to be affected by the influence of gravity, and often topple over during the second stage of drying, leading to non-vertical monolith formation as shown in the lower right image of figure 2. A guide is drawn dividing puddles from monoliths, although the distinction is not absolute. Close to the line, the intermediate structures are more conical in shape, and at high concentrations the monoliths are unstable and tend to grow sideways or fall over. 


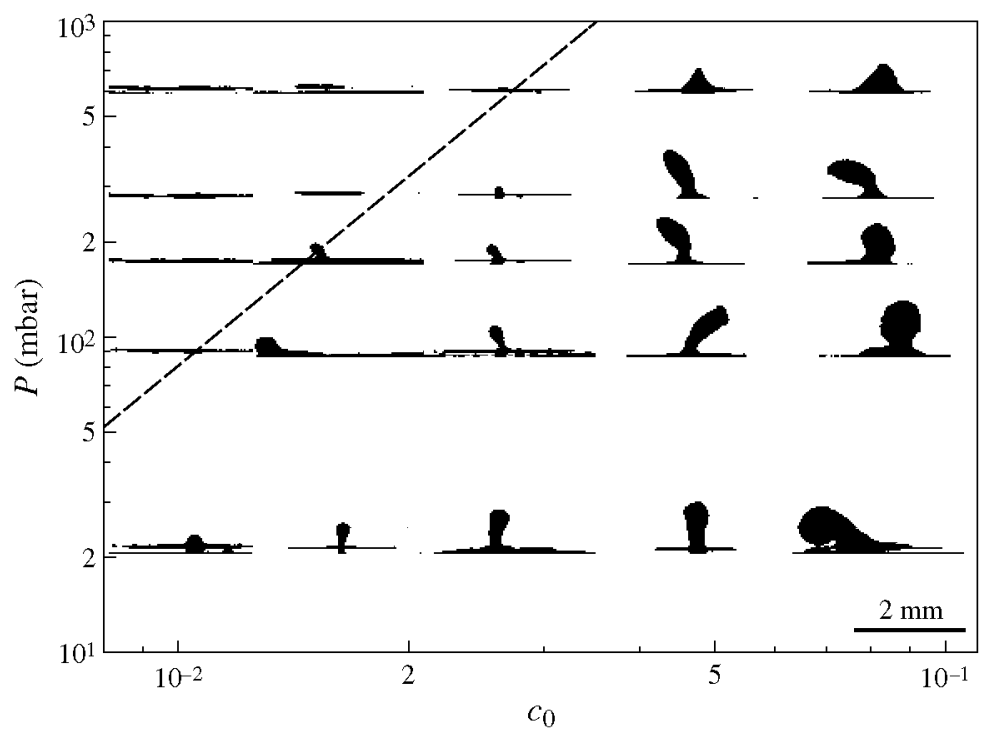

FIGURE 2. The shape of the solid deposit at the end of stage 3 plotted as a function of both initial droplet concentration $c_{0}$ and reduced pressure $P$ on $\log$ axes. The dashed line is a guide to the eye dividing monoliths (below) from flat puddles (above) and has the form $P \sim c_{0}^{2}$. The lower limit of the $y$-axis marks the approximate lower limit of monolith formation, as at pressures below 10 mbar droplets will freeze due to evaporative cooling.

In addition, we have observed coffee-ring stains in some PEO droplets, in which a slightly raised ring of solid polymer is deposited around the initial TL with a very thin crystalline layer in the centre. However, these deposits are only ever seen at very low values of both $c_{0}$ and the initial contact angle, so that by the time the concentration at the edge of the droplet has increased sufficiently for precipitation to begin, there is not enough liquid remaining to be pushed to the centre to form a monolith.

\section{Mathematical model}

We propose a tentative explanation of the trends separating the behaviours of monolith and puddle formation. Let us consider the region near the triple line, as illustrated in figure 3. It is now recognized that for $\theta \ll 90^{\circ}$, the major contribution to evaporation occurs in this zone (Deegan et al. 1997; Hu \& Larson 2002), over the narrow annulus of the liquid/gas interface given by $0<R-r<\epsilon$, where $R$ is contact radius, $r$ is radial distance from the drop centre (assumed axisymmetric) and $\epsilon / R \ll 1$. Length $\epsilon$ is a small, unknown distance, but a reasonable estimate is of order $100 \mathrm{~nm}$ (Askounis et al. 2011). We divide the droplet somewhat arbitrarily into two homogenous regions, a large inner volume $0<r<R-\epsilon$, in which evaporation is negligible and the concentration is assumed to remain at approximately $c_{0}$, and the narrow outer annulus, in which the majority of the evaporation occurs and the polymer concentration will increase. We consider the outer region to have a triangular crosssection of area $(1 / 2) \epsilon^{2} \tan \theta$ and total surface area $2 \pi R \epsilon$. Since we have experimental values of overall evaporation rate, $-\dot{V}$, we may estimate the edge evaporative volume flux, $j$, assumed to be averaged over the surface of the annulus, and to account for a 


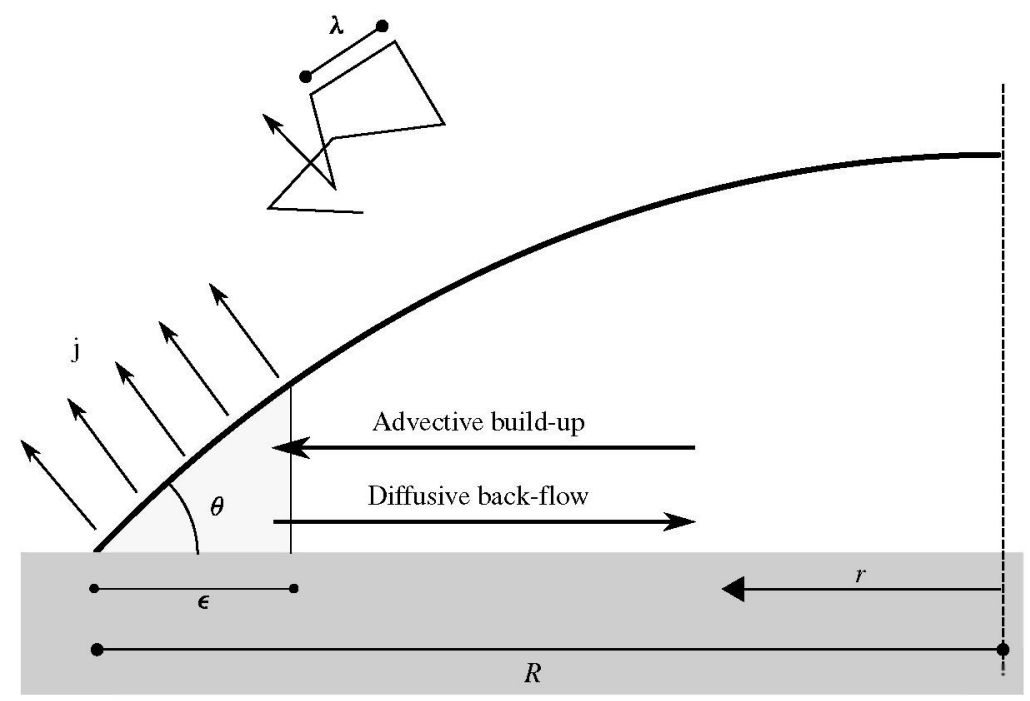

FIGURE 3. Sketch of the droplet geometry used in the mathematical model. The variable $r$ is measured outwards from the centre of the droplet towards the TL at $r=R$. The triangular cross-section annulus, of width $\epsilon$, is exaggerated here for clarity and is assumed to be only a small fraction of $R . \lambda$ is the mean free path in air, and is sufficiently small $(\lambda \ll R)$ for all values of $P$ for evaporation to be diffusion limited.

significant fraction $0 \ll K<1$ of the total evaporation:

$$
j \approx-\frac{K \dot{V}}{2 \pi R \epsilon} .
$$

For the (idealized) quasi-static situation in which the cross-sectional area of the edge region does not vary with time (equivalent to constant contact angle), the water lost by evaporation from this region must be replaced by the arrival of liquid from the centre of the droplet with equal volume. However, unlike the evaporate, the replenishing liquid will not be pure water but will also contain polymer at a concentration $c_{0}$. Following the argument presented in Askounis et al. (2011), we write the total volume of water lost by evaporation per unit length of the TL as $j \epsilon / \cos \theta$, so the incoming volume of polymer per unit length is $c_{0}$ multiplied by this value. The rate of increase in polymer concentration due to advective flux, $\left.(\mathrm{d} c / \mathrm{d} t)\right|_{+}$, is found by dividing by the area of the triangular region, leading to

$$
\left.\frac{\mathrm{d} c}{\mathrm{~d} t}\right|_{+} \approx \frac{2 j c_{0}}{\epsilon \sin \theta} .
$$

This advective build-up of polymer near the TL will, to some extent, be countered by a diffusional back flow due to the developing polymer concentration gradient. For very dilute polymer solutions, the gradient diffusion coefficient is equal to that given by the Stokes-Einstein relation for single-molecule self-diffusion $D$ :

$$
D=\frac{k T}{6 \pi \eta_{L} R_{h}},
$$

where $k, T$ and $\eta_{L}$ are respectively Boltzmann's constant, absolute temperature and solvent viscosity, and $R_{h}$ is the hydrodynamic radius of the polymer. For more 
concentrated, entangled solutions, the gradient diffusion can be faster, as the network entanglement length replaces $R_{h}$ in (4.3) (Doi \& Edwards 1998). Notwithstanding this dependence on concentration, as we do not vary the polymer length, solvent or temperature, molecules in all droplets will have the same limiting value of the gradient diffusion coefficient, which will be some multiple of $D$. In the spirit of our order-of-magnitude calculation, we continue using $D$, but acknowledge that a more rigourous mathematical approach should allow for collective diffusion.

We then use Fick's second law to relate the rate of concentration decrease in the annulus due to diffusion, $\left.(\mathrm{d} c / \mathrm{d} t)\right|_{-}$, to the spatial variation in concentration gradient, $\partial^{2} c / \partial r^{2}$ :

$$
\left.\frac{\partial c}{\partial t}\right|_{-}=D \frac{\partial^{2} c}{\partial r^{2}}
$$

Due to the nature of the advective flow, $\partial c / \partial r$ may be assumed to be of the order of $\left(1-c_{0}\right) / \epsilon$, the concentration gradient across the annulus. As the inner region is homogeneous, the concentration gradient here is virtually 0 , so the required second derivative can be estimated as the difference in the gradients divided by the distance $\epsilon / 2$, which leads to the required expression:

$$
\left.\frac{\partial c}{\partial t}\right|_{-}=D \frac{2\left(1-c_{0}\right)}{\epsilon^{2}}
$$

Thus, from (4.1), (4.2) and (4.5), we may consider the ratio, $\chi$, of (local) concentration build-up due to advection and evaporation on one hand, and concentration reduction due to diffusion on the other:

$$
\chi=\frac{|\mathrm{d} c / \mathrm{d} t|_{+}}{|\partial c / \partial t|_{-}} \approx \frac{K}{2 \pi} \frac{c_{0}}{1-c_{0}} \frac{\dot{V}}{D R \sin \theta} .
$$

The ratio $\chi$ is like a Péclet number for the region near the TL, comparing the relative effects of flux and diffusion. Clearly, if $\chi>1$, net build-up is to be expected near the TL, but, for $\chi<1$, polymer concentration should stay uniform throughout the droplet. With terms $K$ and $D$ assumed constant, it can be seen that the three crucial factors determining $\chi$ are the initial concentration, the droplet shape and the evaporation rate.

In figure 4 , we plot two dimensionless quantities $\dot{V} / D R \sin \theta$ vs $c_{0}\left(1-c_{0}\right)^{-1}$ on log axes, using $D=25 \mu \mathrm{m}^{2} \mathrm{~s}^{-1}$ (Baldwin et al. 2011) (in good agreement with (4.3) using $R_{h}=10 \mathrm{~nm}$ ) and indicate monoliths by crosses and puddles by circles. A straight line corresponding to $2 \pi \chi / K=4$ splits the resulting structures into two families. Setting $K=1$ gives $\chi \approx 1.6$, which is close to 1 , as anticipated. Discrepancies with the data are most likely due to limitations with the theory, which is only valid for $\theta$ intermediate between 0 and $90^{\circ}$ : for low $\theta$ the droplet is more like a drying film in which only vertical, not horizontal, flux is important; on the other hand, close to $90^{\circ}$, flux is uniform across the surface, so the assumption that most evaporation takes place within $\epsilon$ of the TL breaks down.

At this stage, we can only surmise the reason for correlation between monolith formation and $\chi>1$, but a likely explanation seems to be that the latter leads to significant deposition of solid PEO on the substrate following local evaporation near the TL. The drop thus effectively finds itself on a layer of PEO for which the contact angle is much greater, over $90^{\circ}$. Thus, the capillary balance (Young's equation) is modified, causing the liquid front to recede until it reaches the equilibrium contact angle between the polymer solution and the solid PEO layer: the drop effectively 


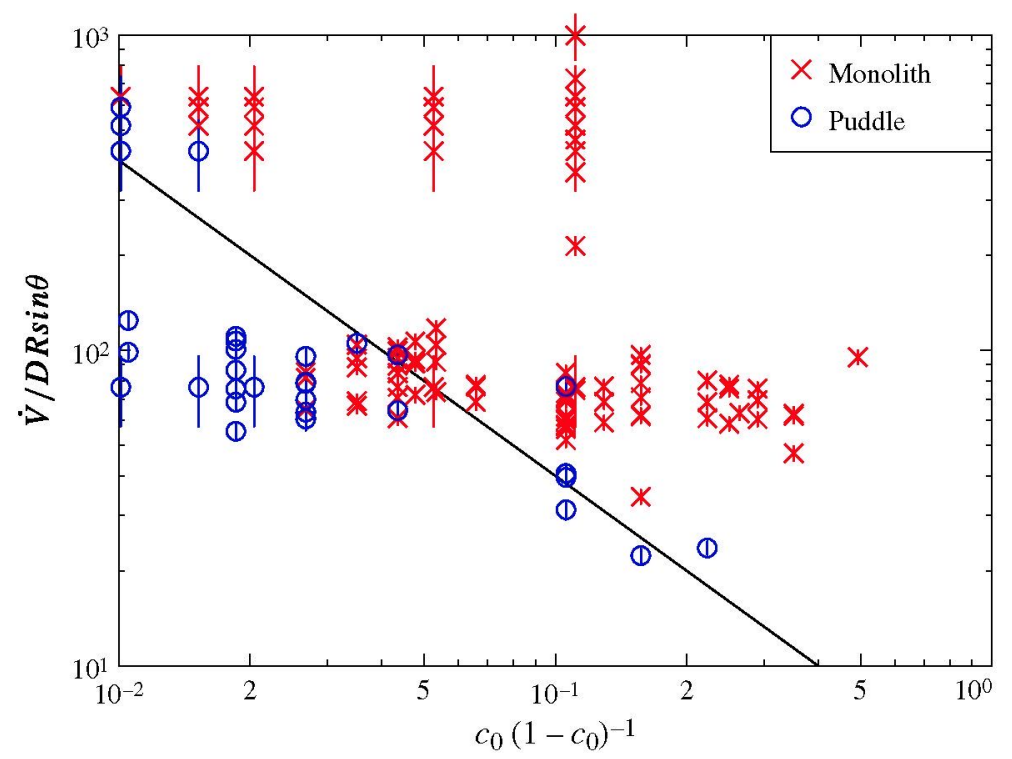

FIGURE 4. (Colour online available at journals.cambridge.org/flm) Phase diagram showing the nature of the final deposit, either a tall central monolith $(x)$ or a flat puddle (o). The vertical axis combines evaporation rate, droplet geometry and diffusion coefficient, as described in the text, and the horizontal axis is a function of initial droplet concentration $c_{0}$. Uncertainties are primarily due to difficulties in measuring evaporation rates when droplets dry rapidly in low-pressure conditions. The solid line is from the theoretical model, and corresponds to a value of $\chi \approx 1.6$. Data points include both previously published data (Baldwin et al. 2011) and approximately 30 new experiments predominantly recorded at low pressures (the upper band of points all above 20 on the vertical axis) along with several other points at higher pressures.

recedes on its own evaporation deposit. When the equilibrium angle has been attained, evaporation continues at constant $\theta$, allowing the monolith to grow. An alternative explanation is that the solid PEO forms a constrictive collar which mechanically squeezes the remaining droplet inwards. The fourth and fifth images for $P=25$ mbar in figure 1 show this solid collar growing at the base of the droplet, but from the images it is impossible to determine causality. Furthermore, at high polymer concentrations chain entanglement may become important: polymer molecules near the TL will tend to become stretched (Abramchuk et al. 2001) and thus the combination of entropic restoring forces and entanglement with chains nearer to the drop centre may tend to draw the peripheral polymer towards the centre.

\section{Conclusions}

In conclusion, our investigation into drying droplets shows that, in stark contrast to the familiar coffee stain in which all material is carried to the droplet edge, aqueous poly(ethylene oxide) solutions deposit nearly all the dissolved material into a tall central monolith. The drying process includes a remarkable 'bootstrap-building' step in which the liquid droplet is lifted above the substrate by the solid precipitate. By increasing the evaporation rate using pressures down to $25 \mathrm{mbar}$, we observed monoliths from droplets with initial concentration as low as $1 \%$. By comparing the opposing effects on polymer concentration due to flux-driven buildup and diffusive 
homogenization, we defined a dimensionless Péclet-type number $\chi$ combining values of flux, diffusion coefficient, droplet geometry and concentration. Plotting experimental values of $\chi$ as a function of $c_{0}$ confirms that monoliths form when $\chi>1$. Further work will investigate in detail the bootstrap-building stage.

\section{Acknowledgements}

K.A.B. is funded by Nottingham Trent University's Vice Chancellor, D.J.F. was supported in part by the Royal Society and by the Nuffield Foundation. S.R. was an ERASMUS exchange student from IUT de Lannion, Université de Rennes, France.

Supplementary movies are available at journals.cambridge.org/flm.

\section{REFERENCES}

Abramchuk, S. S., Khokhlov, A. R., Iwataki, T., OANa, H. \& Yoshikawa, K. 2001 Direct observation of DNA molecules in a convection flow of a drying droplet. Eur. Phys. Lett. 55 (2), 294-300.

Askounis, A., Orejon, D., Koutsos, V., Sefiane, K. \& Shanahan, M. E. R. 2011 Nanoparticle deposits near the contact line of pinned volatile droplets: size and shape revealed by atomic force microscopy. Soft Matt. 7, 4152-4155.

Baldwin, K. A., Granjard, M., Willmer, D. I., Sefiane, K. \& Fairhurst, D. J. 2011 Drying and deposition of poly(ethylene oxide) droplets determined by Peclet number. Soft Matt. 7, 7819-7826.

Cazabat, A.-M. \& Geoffroy, G. 2010 Evaporation of macroscopic sessile droplets. Soft Matt. 6, 2591-2612.

Deegan, R. D., Bakajin, O., Dupont, T. F., Huber, G., Nagel, S. R. \& Witten, T. A. 1997 Capillary flow as the cause of ring stains from dried liquid drops. Nature 389 (6653), 827-829.

DoI, M. \& Edwards, S. F. 1998 The Theory of Polymer Dynamics. Clarendon.

Hammouda, B., Ho, D. L. \& Kline, S. 2004 Insight into clustering in poly(ethylene oxide) solutions. Macromolecules 37 (18), 6932-6937.

HU, H. \& Larson, R. G. 2002 Evaporation of a sessile droplet on a substrate. J. Phys. Chem. B $106(6), 1334-1344$.

HU, H. \& LARSon, R. G. 2006 Marangoni effect reverses coffee-ring depositions. J. Phys. Chem. B 110 (14), 7090-7094.

LI, G. \& GRAF, K. 2009 Microstructures formation by deposition of toluene drops on polystyrene surface. Phys. Chem. Chem. Phys. 11 (33), 7137-7144.

Marín, Á. G., Gelderblom, H., Lohse, D. \& SnoeiJer, J. H. 2011 Order-to-disorder transition in ring-shaped colloidal stains. Phys. Rev. Lett. 107, 085502.

PARISSE, F. \& Allain, C. 1997 Drying of colloidal suspension droplets: experimental study and profile renormalization. Langmuir 13 (14), 3598-3602.

Pauchard, L. \& Allain, C. 2003 Buckling instability induced by polymer solution drying. Eur. Phys. Lett. 62 (6), 897-903.

Rowan, S. M., Newton, M. I., Driewer, F. W. \& McHale, G. 2000 Evaporation of microdroplets of azeotropic liquids. J. Phys. Chem. B 104 (34), 8217-8220.

Stannard, A. 2011 Dewetting-mediated pattern formation in nanoparticle assemblies. $J$. Phys.: Condens. Matter 23, 083001.

Willmer, D., Baldwin, K. A., Kwartnik, C. \& Fairhurst, D. J. 2010 Growth of solid conical structures during multistage drying of sessile poly(ethylene oxide) droplets. Phys. Chem. Chem. Phys. 12 (16), 3998-4004.

Yunker, P. J., Still, T., Lohr, M. A. \& Yodh, A. G. 2011 Suppression of the coffee-ring effect by shape-dependent capillary interactions. Nature 476, 308-311. 